Kong. Res. J. 2(2) : 30-33, 2015

ISSN 2349-2694

Kongunadu Arts and Science College, Coimbatore.

\title{
b-CHROMATIC NUMBER OF CORONA PRODUCT OF CROWN GRAPH AND COMPLETE BIPARTITE GRAPH WITH PATH GRAPH
}

\author{
Vijayalakshmi, D. and G. Mohanappriya \\ Department of Mathematics, Kongunadu Arts \& Science College, Coimbatore-641 029. \\ E.mail: vijikasc@gmail.com; mohanappriyag25@gmail.com
}

\begin{abstract}
A b-coloring of a graph is a proper coloring where each color admits at least one node (called dominating node) adjacent to every other used color. The maximum number of colors needed to b-color a graph $\mathrm{G}$ is called the b-chromatic number and is denoted by $\varphi(G)$. In this paper, we find the b-chromatic number and some of the structural properties of corona product of crown graph and complete bipartite graph with path graph.
\end{abstract}

Keywords: Corona product, crown graph, complete bipartite graph, path graph.

\section{INTRODUCTION}

A b-coloring by k-colors is a proper coloring of the vertices of graph $\mathrm{G}$ such that in each color classes there exists a vertex that has neighbors in all the other $\mathrm{k}-1$ color classes. The b-chromatic number $\varphi(\mathrm{G})$ is the largest number $\mathrm{k}$ for which $\mathrm{G}$ admits a bcoloring with k-colors (Irving and Manlove, 1999).

The corona G1 $\circ \mathrm{G} 2$ of two graphs G1 and G2 is defined as a graph obtained by taking one copy of G1 (which has p1 vertices) and p1 copies of G2 and attach one copy of G2 at every vertex of G1 (Harary, 1972).

In this paper we find for which the largest number $\mathrm{k}$ for which corona product of crown graph and complete bipartite graph with path graph admits a b-coloring with k-colors. And also we find some of its structural properties (Venkatachalam and Vernold Vivin, 2010; Vernold Vivin and Venkatachalam, 2012; Vijayalakshmi and Thilagavathi, 2012)

\section{Definition}

\subsection{Crown Graph}

A crown graph on $2 \mathrm{n}$ vertices is an undirected graph with two sets of vertices $u_{i}$ and $v_{i}$ and with an edge from $u_{i}$ to $v_{j}$ whenever $i \neq j$. The crown graph can be viewed as a complete bipartite graph from which the edges of a perfect matching have been removed (Wikipedia).

\subsection{Complete Bipartite Graph}

A complete bipartite graph is a graph whose vertices can be partitioned into two subsets $V_{1}$ and
$V_{2}$ such that no edge has both endpoints in the same subset, and every possible edge that could connect vertices in different subsets is part of the graph. That is, it is a bipartite graph $\left(V_{1}, V_{2}, E\right)$ such that for every two vertices $v_{1} \in V_{1}$ and $v_{2} \in V_{2}, v_{1} v_{2}$ is an edge in $E$. A complete bipartite graph with partitions of size $\left|V_{1}\right|=m$ and $\left|V_{2}\right|=n$, is denoted $K_{\mathrm{m}, \mathrm{n}}$ (Balakrishnan, 2004; Balakrishnan and Ranganathan, 2012).

\subsection{Fan Graph}

and A Fan graph $F_{m, n}$ is defined as the graph join $K_{m}$, where $K_{m}$ the empty graph on nodes is and $\mathrm{P}_{\mathrm{n}}$ is the path on $\mathrm{n}$ nodes (Wikipedia).

\subsection{Path Graph}

The path graph $\mathrm{P}_{\mathrm{n}}$ is a tree with two nodes of vertex degree 1 , and the other $\mathrm{n}-2$ nodes of vertex degree (Harary, 1972).

\subsection{Corona Product}

Corona product or simply corona of any graph G1 and graph G2, defined as the graph which is the disjoint union of one copy of G1 and |V1| copies of G2 (|V1| is the number of vertices of G1) in which each vertex of the copy of G1 is connected to all vertices of a separate copy of G2 (Harary, 1972).

\section{6. b-coloring}

A b-coloring of a graph is a proper coloring such that every color class contains a vertex that is adjacent to all other color classes. The b-chromatic number of a graph $G$, denoted by $\varphi(G)$, is the maximum number $t$ such that $G$ admits a b-coloring with t colors (Irving and Manlove, 1999). 


\section{CORONA PRODUCT OF CROWN GRAPH WITH PATH GRAPH}

3.1. b-chromatic number of corona product of Crown Graph with Path Graph

\subsubsection{Theorem}

For any $\mathrm{n} \geq 3, \varphi\left[S_{n \eta}^{\circ \circ} P\right]=2 \mathrm{n}$.

Proof: Let $S^{0}$ be any Crown graph with vertices, $\mathrm{V}=$ $\left\{v_{1}, v_{2}, \ldots, v_{n}\right\}^{y}$ and $V=\left\{v_{1}, v_{1}, \ldots, v^{\prime}\right\}$ i.e. $\mathrm{V}\left(S^{0}\right)=\mathrm{V} \mathrm{U}$ $V$. Let the edges of $L_{n}$ be $\mathrm{E}\left(S_{n}^{0}\right)=\left\{e_{j}: 1 \leq j \leq n^{2}-\right.$ $n$ ) where $e_{j}$ is the edge connecting $v_{i}$ and $v$, foreveryi $\neq j$.

Let $P_{n}$ be ant path graph of length n-1 with n-vertices. $\mathrm{V}\left(p^{\prime}{ }_{n}\right)=\left\{u_{\mathrm{ij}}: 1 \leq i \leq 2 n, 1 \leq l \leq n, 1 \leq\right.$ $j \leq n\}$ and and $E P_{n}$ be $\left\{\mathrm{e}_{\mathrm{pi}}: 2 \mathrm{n}-1 \leq \mathrm{i} \leq \mathrm{n}-1\right\}$.

By the definition of corona graph each vertex in $\mathrm{S}_{\mathrm{n}}^{0}$ is adjacent to every vertex copy of $P_{n}$, i.e. vertices of $V\left(L_{n} \circ P_{n}\right)=\mathrm{V}\left(S^{0}{ }_{n}\right) U V\left(P_{n}\right)$. Let $\mathrm{E}\left[S_{n}^{0} \circ P_{n}\right]$ be $\mathrm{E}\left(S_{n}^{0}\right) \mathrm{U} E P_{n} \mathrm{U}\left\{\mathrm{e}_{\mathrm{i}}: n^{2}-\mathrm{n}+1 \leq \mathrm{i} \leq\right.$ $\left.5 n^{2}-3 n\right\}$.

Consider the color class $\mathrm{C}=$ $\left\{C_{1}, C_{2}, C_{3}, \ldots, C_{n}, C_{n+1}, C_{n+2}, \ldots, C_{2 n}\right\}$ to color the

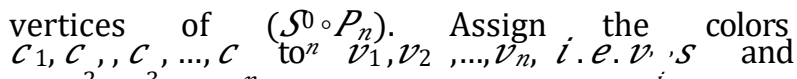
$C_{n+1}, C_{n+2}, \ldots, C_{2 n}$ to $v_{j} \mathrm{~s}, \mathrm{j}=1,2,3, \ldots, \mathrm{n}$ respectively for every $i \neq j, i, j=1,2, \ldots, n$.

From the figure we see that, each $v$ ', $s$ are adjacent to every $v_{i}, S$ for every i not equal to j and vice versa. Hence both $v_{i}^{\prime} S$ and $v_{j}^{\prime} s$ earns its adjacent color for every $\mathrm{i} \neq \mathrm{j}$. To make the aboye coloring to be b-chromatic proper coloring of $\mathrm{V}^{\prime}\left(p^{\prime}\right)_{n}$ by corresponding non-adjacent vertices of its $v_{i}$ 's or $\nu$ '' $S$ respectively. Thus each color has the neighbour

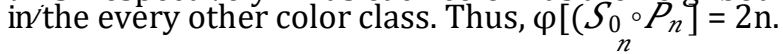

Let us assume that $\varphi\left[S_{0}^{0}{ }_{n} P\right]_{\lambda} 2 n$, let it be $\varphi\left[S_{n} \circ P_{n}\right]=2 \mathrm{n}+1$. The graph $S_{n} \circ P_{n}$ must requires $2 n+2$ vertices of degree $2 n+1$, all with distinct color and each must have adjacent with all of the other color class, but at least one color class which does

not have a color dominating vertex in $\left[S_{n}^{0} \circ P_{n}\right]$, which invalidates the definition of $b$-coloring. Hence, $\varphi\left[S_{n}^{0} \circ P_{n}\right]$ not equal to $2 \mathrm{n}+1$, it must be less than $2 \mathrm{n}+1$ i.e. $\varphi\left[S_{n} \circ P_{n}\right]=2 \mathrm{n}$. Thus, for any $\mathrm{n} \geq 3$, the $\mathrm{b}$ chromatic number of corona graph of crown graph with path graph is $2 \mathrm{n}$.

\subsection{Illustration: b-coloring of corona productof Crown Graph with Path Graph}

\subsubsection{Theorem}

For any $\mathrm{n} \geq 3, \mathrm{q}\left[S_{n}^{0} \circ P_{n}\right]=5 n^{2}-3 \mathrm{n}$

Proof: q[ $\left(S_{n}^{0} \circ P_{n}\right]=$ Number of edges in $S^{0}+{ }_{n} 2 \mathrm{n} \mathrm{x}$ Number of edges in $F_{n}$

$$
\begin{aligned}
& =n^{2}-n+2 \mathrm{n}(2 \mathrm{n}-1) \\
& =n^{2}-n+4 n^{2}-2 \mathrm{n}-1 . \\
& =5 n^{2}-3 n
\end{aligned}
$$

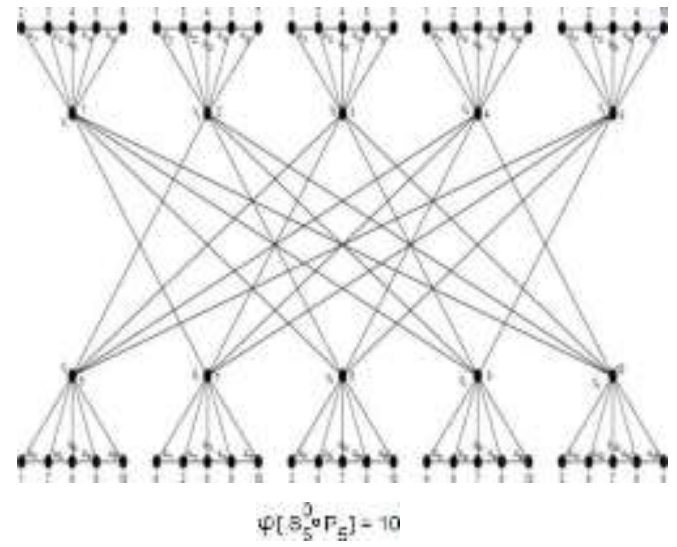

\subsubsection{Theorem}

For any $\mathrm{n} \geq 3$, the vertex polynomial of $\left(S_{n} 0 \circ P_{n}\right)$ be $4 n x^{2}+2 n^{2}-4 n x^{3}+4 x^{n+2}$

Proof: $\mathrm{V}\left(\left(S_{n}^{0} \circ P_{n} ; \mathrm{x}\right)=\stackrel{\Delta(\mathrm{G})}{k} \bigvee_{1} \mathrm{x}^{\mathrm{k}}\right.$

$=$ No of vertices having degree $2 \times \mathrm{x}_{3}^{2}+$

No of vertices having degree $3 \times x+$

No of vertices having degree $n+2 \times x^{n+2}$

\begin{tabular}{|c|c|c|c|c|c|}
\hline $\begin{array}{c}\text { Propert } \\
\text { ies } \\
\text { Graphs }\end{array}$ & $\begin{array}{l}\text { No. of } \\
\text { Vertex }\end{array}$ & $\begin{array}{l}\text { No. of } \\
\text { Edges }\end{array}$ & $\begin{array}{c}\text { Maxim } \\
\text { um } \\
\text { Degree }\end{array}$ & $\underset{\text { um }}{\text { Minim }}$ & $\begin{array}{c}\text { Vertex } \\
\text { Polynomi } \\
\text { al }\end{array}$ \\
\hline $\begin{array}{l}\text { Path } \\
\text { Graph }\end{array}$ & $\mathrm{n}$ & $\mathrm{n}-1$ & 2 & 1 & $\begin{array}{l}2 \mathrm{x}+(\mathrm{n}- \\
\left(n^{2}\right) x^{2}\end{array}$ \\
\hline $\begin{array}{l}\text { Crown } \\
\text { Graph }\end{array}$ & $2 n$ & $\stackrel{2}{n-\mathrm{n}}$ & $n-1$ & $n-1$ & $\overline{4} n \mathfrak{x}_{2} x^{n-1}$ \\
\hline $\left.\begin{array}{c}\left(\mathcal{P}_{n}^{0} \circ\right. \\
n\end{array}\right)$ & $\begin{array}{c}2 n(n+ \\
1)\end{array}$ & $\begin{array}{c}5 n^{2}- \\
3 n\end{array}$ & $2 n-1$ & 2 & $\begin{array}{l} \pm(2 n)^{2} \mathfrak{X} \\
+(2 n) x^{n+}\end{array}$ \\
\hline
\end{tabular}

$$
=4 n \mathrm{x}^{2}+2 \mathrm{n}^{2}-4 \mathrm{nx}^{3}+2 \mathrm{nx}^{\mathrm{n}+2} \text {. }
$$

3.2.3. Some Structural Properties of $\left(S_{n}^{0} \circ P_{n}\right) n \geq 3$.

\section{CORONA PRODUCT OF COMPLETE BIPARTITE GRAPH WITH PATH GRAPH}

4.1. b-chromatic number on Corona Product of Complete Bipartite Graph with Path Graph 


\subsubsection{Theorem}

$\begin{array}{ll}\text { For any } \mathrm{n} \geq 3, \varphi & \circ K_{m, n}\end{array} \quad\left\{\begin{array}{ll}{[\mathrm{n}+1} & \mathrm{m}>\mathrm{n} \\ 2 \mathrm{n}-1 & \mathrm{~m}<\mathrm{n}\end{array} \quad \mathrm{m}=\mathrm{n}\right.$

Proof: Let $K_{m, n}$ be any complete bipartite graph with vertices, $V=\left\{\mathcal{V}_{1}, \mathcal{V}_{2}, \ldots, \mathcal{V}_{n}\right\}$ and $V=\left\{\mathcal{V}_{1}, v_{2}, \ldots, \mathcal{V}_{n}\right\}$ i.e. $\mathrm{V}\left(K_{m, n}\right)=\mathrm{V}$ U $V$. Let the edges $K_{m, n}$ of be $\mathrm{E}\left(K_{m, n}\right)=\left\{e_{j}: 1 \leq j \leq n^{2}\right\}$ where $e_{j}$ is the edge connecting $v_{i}$ and $v$.

Let $P$ be ant path graph of length n-1 with n-vertices. $\mathrm{V}\left(p_{\eta}^{\prime}\right)=\left\{u_{\mathrm{ij}:} 1 \leq i \leq 2 n, 1 \leq l \leq n, 1 \leq\right.$ $j \leq n\}$ and $E P_{n}$ be $\left\{\mathrm{e}_{\mathrm{pi}}: 2 \mathrm{n}-1 \leq \mathrm{i} \leq \mathrm{n}-1\right\}$

By the definition of corona graph each vertex in $\mathrm{K}_{\mathrm{m}, \mathrm{n}}$ is adjacent to every vertex copy of $P_{n}$, i.e. vertices of $V\left(K_{m, n^{\circ}} P_{n}\right)=\mathrm{V}\left(K_{m, n}\right) U V\left(P_{n}\right)$.

Let the edges of $K_{m, n}{ }^{\circ} P_{n}$ be $\mathrm{E}[K \circ P]=\mathrm{E}(K) \cup \in P U\left\{\mathrm{e}: n^{2}+1 \leq \mathrm{i} \leq\right.$

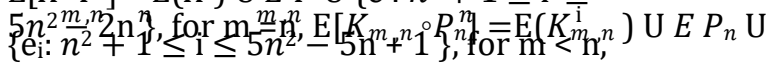
$\mathrm{E}\left[K_{m, n} \circ P_{n}\right]=\mathrm{E}\left(K_{m, n}\right) \quad \mathrm{U} \quad E P_{n} \mathrm{U} \quad\left\{\mathrm{e}_{\mathrm{i}}: n^{2}+1 \leq \mathrm{i} \leq\right.$ $\left.5 n^{2}+n-1\right\}$, for $m>n$.

Consider the color class $\mathrm{C}=$

$\left\{c_{1}, c_{2}, c_{3}, \ldots, c_{n}, c_{n+1}, c_{n+2}, \ldots, c_{2 n+1}\right\}$ to color the vertices of $\left(K_{m, n} \circ P_{n}\right)$. The proof follows from the following cases.

Case (i) $m=n$

\{

Consider the color class $C=$

1

$\left.c_{1}, c_{2}, c_{3}, \ldots, c_{n}, c_{n+1}, c_{n+2}, \ldots, c_{2 n}\right\}$ to color the vertices of $K\left(m, n \circ P_{n}\right), m=n$. Assign the colors

$c_{1}, c_{2}, c_{3}, \ldots, c_{n}$ to $v_{1}, v_{2}, \ldots, v_{n}$, i.e. $v$ i 's and

$c_{n+1}, c_{n+2}, \ldots, c_{m}$ tov $_{j} \mathrm{~s}, \mathrm{j}=1,2,3, \ldots, \mathrm{m}$ respectively.

From the figure we assure that, each $v$, s $i$

are adjacent to every $v_{j},{ }_{s}^{\prime}$ for and vice versa. Hence both $v$ ' 's and $v$ ', $s$ earns its adjacent color. To make

the above coloring to be b-chromatic proper coloring of $\mathrm{V}\left(p_{n}^{l}\right)$ by corresponding non-adjacent vertices of its $v_{i}$ 's or $v_{i}$ 's respectively, and the remaining vertices are colored properly by the colors in the color class. Thus each color has the neighbor in the every other color class. Thus, $\varphi\left[K_{m, n}{ }^{\circ} P_{n}\right]=2 \mathrm{n}$.

Let us assume that $\varphi\left[\left(K_{m, n}{ }^{\circ} P_{n}\right]>2\right.$ n, say $\varphi\left[K_{m, n} \circ P_{n}\right]=2 \mathrm{n}+1$. The graph $\left[K_{m, n} \circ P_{n}\right]$ must requires $2 n+2$ vertices of degree $2 n+1$, all with distinct color and each must have adjacent with all of the other color class which is not possible, since maximum degree of $K_{m, n} \circ P_{n}$ is $2 \mathrm{n}$, hence at least one color class does not have the color dominating vertex, which contradicts the definition of $b$-coloring. Hence, $\varphi\left[K_{m, n}{ }^{\circ} P_{n}\right]$ not equal to $2 \mathrm{n}+1$, must be less than $2 \mathrm{n}+1$ i.e. $\varphi\left[K_{m, n} \circ P_{n}\right]=2 \mathrm{n}$. Thus, for any $\mathrm{n} \geq 3$, the $b$-chromatic number of corona graph of complete bipartite graph with path graph is $2 \mathrm{n}$ for each $\mathrm{m}=\mathrm{n}$.

Case (ii) $m>n$

Consider the color class $\mathrm{C}_{2}=$ $\left\{c_{1}, c_{2}, c_{3}, \ldots, c_{n}, c_{n+1}, c_{n+2}, \ldots, c_{2 n+1}\right\}$ to color the

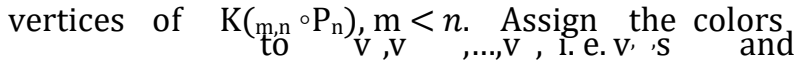
$\mathrm{C}_{1}, \mathrm{C}_{2}, \mathrm{C}_{3}, \ldots, \mathrm{C}_{\mathrm{n}} \quad 12 \mathrm{n} \quad \mathrm{i}$ $c_{n+1}, c_{n+2}, \ldots, c_{2 n+1}$ tov, $j s \quad, j=1,2,3, \ldots, m$ respectively.

The remaining proof of the theorem follows immediately from case (i). Hence $\left[K_{m, n} \circ P_{n}\right]=2 n+1, m>n$.

Case (iii) $m<n$

Consider the color class $C^{\prime}=$

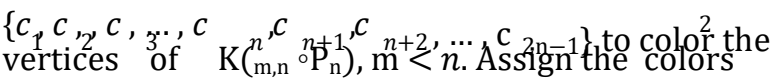
$\mathrm{c}_{1}, \mathrm{c}_{2}, \mathrm{c}_{3}, \ldots, \mathrm{c}_{\mathrm{n}}$ to $\mathrm{v}_{1}, \mathrm{v}_{2}, \ldots, \mathrm{v}_{\mathrm{n}}$, i. e.v', s and $\mathrm{c}_{\mathrm{n}+1}, \mathrm{c}_{\mathrm{n}+2}, \ldots, \mathrm{c}_{2 \mathrm{n}-1}$ tov $^{\prime \prime}{ }_{\mathrm{j}} \mathrm{s}, \mathrm{j}=1,2,3, \ldots, \mathrm{m}$ respectively.

The remaining proof of the theorem follows immediately from case (i). Hence $\left[K_{m, n} \circ P_{n}\right]=2 n-1, m<n$. Hence theproof.

\subsubsection{Illustration Corona Product of Complete Bipartite Graph with Path Graph \\ 4.1.2. Theorem}

For any $m, n \geq 3, q\left[\left(K_{m, n} \circ P_{n}\right)\right]=2 n^{2}+3 m n-m-n$. Proof: $\mathrm{q}\left[\left(\mathrm{S}_{\mathrm{n}}^{0} \circ \mathrm{P}\right]=\right.$ Number of edges in $\mathrm{K}+2 \mathrm{nx}$ Number of edges in Fan graph $F_{n}$

$$
\begin{aligned}
& =m n+(m+n)(2 n-1) \\
& =m n+2 m n-m+2 n^{2}-n .
\end{aligned}
$$

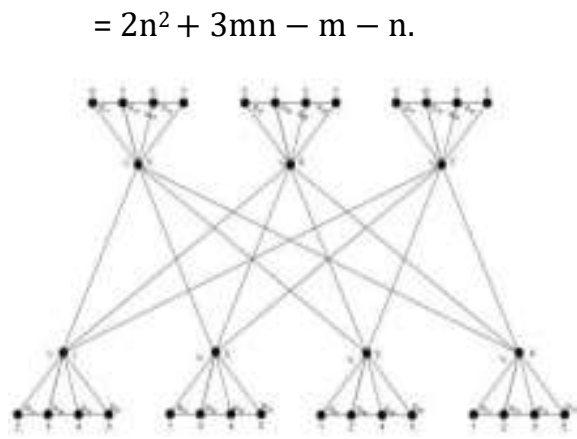

$$
\varphi\left[K_{3,4}, P_{4}\right]=7
$$




\subsubsection{Theorem}

For any $m, n \geq 3$, the vertex polynomial of $\operatorname{be}\left(\mathrm{K}_{\mathrm{m}, \mathrm{n}} \circ \mathrm{P}_{\mathrm{n}}\right) 4 n x^{2}+\left(2 n^{2}-4 n\right) x^{3}+2 n x^{2 n}, \mathrm{~m}=\mathrm{n}$.

$$
\text { Proof: } \underset{\mathrm{m}, \mathrm{n}}{(\mathrm{K}} \stackrel{\mathrm{P}}{\mathrm{n}} ; \mathrm{x})=\stackrel{\Delta(\mathrm{G})}{\mathrm{V}} \mathrm{x}^{\mathrm{k}}
$$

$=$ No of vertices having degree $2 \times \mathrm{x}^{2}+$

No of vertices having degree $3 \times \mathrm{x}^{3}+$

No of vertices having degree $2 \mathrm{n} \times \mathrm{x}^{2 \mathrm{n}}+$

$$
=4 n \mathrm{x}^{2}+2 \mathrm{n}^{2}-4 n \mathrm{x}^{3}+2 n \mathrm{x}^{\mathrm{n}+2} \text {. }
$$

For any $m, n \geq 3$, the vertex polynomial of be $\left(\mathrm{K}_{\mathrm{m}, \mathrm{n}} \circ \mathrm{P}_{\mathrm{n}}\right) 4 n x^{2}+\left(2 n^{2}-4 n\right) x^{3}+2 n x^{2 n}, \mathrm{~m}>\mathrm{n}$.

Proof: $\mathrm{V}\left(\mathrm{K}_{\mathrm{m}, \mathrm{n}} \circ \mathrm{P} ; \mathrm{n}\right)=\Delta(\mathrm{G}) \mathrm{V} \mathrm{x}_{k=1 \mathrm{k}}^{\mathrm{k}}$

$=$ No of vertices having degree $2 \times x^{2}+$

No of vertices having degree $3 \times \mathrm{x}^{3}+$

No of vertices having degree $2 n-1 \times x^{2 n+1}$

$(2 n+1) x^{2 n+1}$.

\subsubsection{Theorem}

\begin{tabular}{|c|c|c|c|c|c|}
\hline $\begin{array}{c}\text { Properties } \\
\text { Graphs }\end{array}$ & $\begin{array}{l}\text { Number of } \\
\text { Vertex }\end{array}$ & $\begin{array}{l}\text { Number of } \\
\text { Edges }\end{array}$ & $\begin{array}{c}\text { Maximum } \\
\text { Degree }\end{array}$ & $\begin{array}{c}\text { Minimum } \\
\text { Degree }\end{array}$ & Vertex Polynomial \\
\hline Path Graph & $\mathrm{n}$ & $\mathrm{n}-1$ & 2 & 1 & $2 \mathrm{x}+(\mathrm{n}-2) x^{2}$ \\
\hline Complete & $m+n$ & $n^{2}$ & $\max \{m, n\}$ & $\min \{m, n\}$ & $(2 n) x^{n}$ \\
\hline $\begin{array}{l}\text { Bipartite Graph } \\
\left(K_{m, n} \circ P_{n}\right) \\
\mathrm{m}=\mathrm{n}\end{array}$ & $2 n^{2}+2 n$ & $5 n^{2}-2 n$ & $2 n$ & 2 & $\begin{array}{r}4 n x^{2}+\left(2 n^{2}-4 n\right) x^{3} \\
+2 n x^{2} n\end{array}$ \\
\hline $\begin{array}{l}\left(K_{m, n} \circ P_{n}\right) \\
\mathrm{m}<\mathrm{n}\end{array}$ & $\begin{aligned} & 2 n^{2} \\
+ & n-1\end{aligned}$ & $\begin{array}{r}5 n^{2} \\
-5 n+1\end{array}$ & $2 n$ & 2 & $\begin{array}{r}(4 n-2) x^{2}+\left(2 n^{2}-5 n\right. \\
+2) x^{3}\end{array}$ \\
\hline $\begin{array}{l}\left(K_{m, n} \circ P_{n}\right) \\
\mathrm{m}>\mathrm{n}\end{array}$ & $\begin{array}{r}2 n^{2} \\
+3 n+1\end{array}$ & $\begin{aligned} & 5 n^{2} \\
+ & n-1\end{aligned}$ & $2 n+1$ & 2 & $\begin{array}{r}+n x^{2 n-1} \\
+(n \\
-1) x^{2 n} \\
(4 n+2) x^{2} \\
+\left(2 n^{2}-3 n-2\right) x^{3} \\
+(2 n+1) x^{2 n+1}\end{array}$ \\
\hline
\end{tabular}

4.4.5. Some Structural Properties of $\left(K_{m, n} \circ P_{n}\right), n \geq 3$.

\section{CONCLUSION}

In this paper we operated the graph operation corona product on crown graph and complete bipartite graph with path graph, we get corona product of crown graph with path graph and corona product of complete bipartite graph with path graph and also we find its b-chromatic number and some of its structural properties.

\section{REFERENCES}

Balakrishnan, R. and K. Ranganathan, (2012). A textbook of Graph Theory. Springer, New York.

Balakrishnan, V.K. (2004). Graph Theory. Tata McGraw-Hill Publishing Ltd, New Delhi.

Harary, F. (1972). Graph Theory. Addison-Wesley, Reading, Massachusetts.
Irving, R.W. and D.F. Manlove, (1999). The bchromatic number of a graph. Discrete. Appl. Math., 91: 127-141.

Venkatachalam, M. and J. Vernold Vivin, (2010). The b-chromatic number of star graph families. $L e$ Matematica, 65(1): 119-125.

Vernold Vivin, J. and M. Venkatachalam, (2012). The b-Chromatic Number of Corona Graphs, Utilitas Mathematica, 88: 299-307.

Vijayalakshmi, D. and K. Thilagavathi, (2012). Study On b-Chromatic Coloring of Some Graphs. Ph.D., Thesis, Bharathiar University.

Wikipedia, the free Encyclopedia. 